\title{
Experimental Comparison of 2D and 3D Technology Mediated Paramedic - Physician Collaboration in Remote Emergency Medical Situations
}

\author{
Diane H. Sonnenwald, ${ }^{1}$ Hanna Maurin \\ Göteborg University and University College of Borås, Sweden \\ Bruce Cairns, James E. Manning \\ University of North Carolina, USA \\ Eugene B. Freid \\ Nemours Children's Clinic, USA \\ Greg Welch, Henry Fuchs \\ University of North Carolina, USA
}

\begin{abstract}
We are investigating the potential of 3D telepresence technology to support collaboration among geographically separated medical personnel in trauma emergency care situations. 3D telepresence technology has the potential to provide richer visual information than current $2 \mathrm{D}$ video conferencing techniques. This may be of benefit in diagnosing and treating patients in emergency situations where specialized medical expertise is not locally available. We conducted an experimental evaluation, simulating an emergency medical situation and examining the interaction between the attending paramedic and remote, consulting physician. Post-questionnaire data illustrate that the information provided by the consulting physician was perceived to be more useful by the paramedic in the 3D condition than the $2 \mathrm{D}$ condition. However, the data pertaining to the quality of interaction and trust between the consulting physician and paramedic showed mixed results. The implications of these results are discussed.

\section{Introduction}

Trauma is a significant health problem, frequently referred to as the 'hidden epidemic of modern society' because it is responsible for more productive years lost than heart disease, cancer and stroke combined (Meyer, 1998; Coates \& Goode, 2001). 3D telepresence technology, an emergent information and communications technology that may ultimately provide dynamic 3D views of remote scenes, has the potential to provide richer visual information than current 2D video conferencing techniques and increase a physician's knowledge about a remote patient, the trauma scene, and actions performed by remote medical personnel. Using 3D technology may provide people in rural, catastrophic and hazardous areas, as well as disabled and elderly people who can not be easily transported, access to experts at large medical care facilities.
\end{abstract}

As indicated in the National Academy's report on telemedicine evaluation (Field, 1996), it is of critical importance to examine the acceptability and practicality of technology in medicine. Today only prototype demonstrations of the 3D telepresence technology exist; the technology is not sufficiently developed to be evaluated using standard usability methods. However, research funding agencies would like to know today whether the technology has potential to improve health care in emergency medical care situations. The funding agencies would like to avoid spend millions of dollars developing yet another technology that does not have a positive impact on medical care. This poses a complex challenge. How can we evaluate the potential impact of a technology within complex, dynamic work contexts when the technology does not yet exist?

${ }^{1}$ All correspondence should be addressed to Diane H. Sonnenwald (Mail address: University College of Borås, SE-51090 Borås, Sweden. E- mail address: Diane.Sonnenwald@hb.se.)

Published in: A. Grove (Ed.), Proceedings of the 2006 American Society of Information

Science \& Technology Conference. Washington, DC: American Society for Information

Science and Technology. 
To address this challenge we conducted an experimental evaluation that compared collaboration between paramedics and remote physicians under two conditions. One condition utilized high quality 2D video to support collaboration during a simulated emergency medical situation. The other condition utilized a 3D telepresence proxy. In each condition the same emergency medical situation was simulated. In the simulation a professional paramedic was asked to diagnose and treat a trauma victim. A remote physician was available to assist the paramedic. Performance and post-test data were collected and analyzed to compare the impact of technology in each condition.

This paper discusses the results concerning the collaboration between the paramedics and consulting physician. In particular, usefulness of information, quality of interaction and trust are examined. Results indicate that the information provided by the consulting physician was perceived to be more useful by the paramedic participating in the 3D condition than the 2D condition. However, the data pertaining to the quality of interaction and trust between the consulting physician and attending paramedic showed mixed results. The implications of these results are discussed.

\section{Experimental Evaluation Design}

Evaluating technology for remote consultation in emergency medical situations has unique challenges, many of which can be attributed to the complex context in which emergency situations occur. Patient healthcare priorities, patient privacy, and the dynamics of emergency care in the field make it extremely difficult, if not impossible, to collect evaluation data in the field. Furthermore, collaboration between paramedics in the field and emergency care physicians today is extremely unpredictable. These conditions make observational field studies impractical.

Thus we conducted a controlled lab study using a post-test design. This approach to evaluation has been successfully used in previous research (e.g., Sonnenwald, Whitton, \& Maglaughlin, 2003). When designing the controlled study, we developed hypotheses based on previous research, simulated a realistic medical scenario and tasks, and employed rigorously developed measures to help insure the validity and reliability of the evaluation results without impacting patient care, an important consideration in medicine.

\section{Hypotheses}

Previous research in computer supported cooperative work (e.g., Dourish, Adler, Bellotti, \& Henderson, 1996; Olson \& Olson, 2000) and theory of language (Clark, 1996) suggests that working remotely using $2 \mathrm{D}$ video-conferencing lacks the richness of collocation and face-to-face interaction. In particular it lacks multiple and redundant communication channels, implicit cues, spatial co-references that are difficult to support via computer-mediated communication. This lack of richness is thought to impair collaboration because it is more difficult to establish the common ground that enables individuals to understand the meaning of each other's utterances. Other research (e.g., Starr \& Ruhleder, 1996; Orlikowski, 1993; Olson \& Teasley, 1996) suggests that working remotely may not be compatible with existing work practices. However 3D telepresence technology may facilitate richer interaction between paramedics and remote consulting physicians than currently provided via 2D video-conferencing technology. Therefore, our hypotheses are:

H1: Paramedics will perceive the usefulness of information provided by the consulting physician as greater in the $3 D$ condition than in the $2 D$ condition.

H2: Paramedics will rate the quality of interaction with the consulting physician higher in the $3 D$ condition than in the $2 D$ condition.

H3: Paramedics will report greater levels of trust with respect to the consulting physician in the 3D condition compared to the $2 D$ condition.

\section{Simulation of an emergency medical situation}

In the U.S. paramedics (EMT-P job classification) are typically the first and only medical personnel who 
travel to the scene of medical emergencies. At the scene their job is to diagnose patients, perform all necessary medical procedures they are legally allowed to perform, and when necessary, transport patients to the nearest available and appropriate medical facility. They typically have no prior knowledge about the patient and very limited knowledge about the accident. They often must work quickly and accurately to save lives, and they must do this wherever the patient is located, e.g., in a ditch next to a highway at night when it's raining. It is not possible to evaluate emergent technology under these field conditions. Therefore, we created a simulation of an emergency medical situation in our lab. In particular, we simulated a car accident scene where a male adult, age 38, was found thrown from a car and moaning.

A computerized mannequin, the METI Human Patient Simulator ${ }^{\mathrm{TM}}$, was used to simulate the trauma patient. This state-of-the-art mannequin can be programmed to act and respond in a life-like manner. For example, its pupils dilute in response to light, its chest rises and falls when breathing, its heart rate, breathing pattern and oxygen blood levels respond to drug injections and medical procedures.

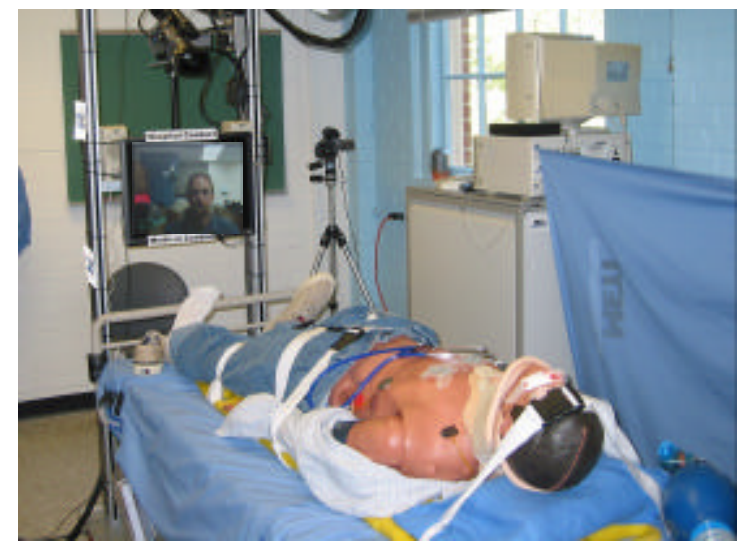

Figure 1. The human patient simulator staged as a car accident victim

The simulated scenario was developed in collaboration with three physicians with different specialities and subsequently tested and refined by five paramedics in a pilot study. We used a darkened room with spotlights and a rotating beacon to simulate an accident scene late in the evening with streetlights and the presence of an ambulance (Figure 1). To increase the fidelity we added background sounds that included traffic noises, people's voices and emergency vehicle sirens.

One key aspect of the simulation design was choosing an appropriate trauma emergency medical task for the participating paramedics to perform. The task needed to have a certain level of complexity, consisting of demanding medical decision-making and technical skills. It needed to be a task paramedics were qualified to perform with measurable outcomes, and a task they would seek advice on. In addition the task needed to be socially relevant - an important medical problem.

The task selected was the diagnosis and treatment of a difficult airway, including performing a surgical cricothyrotomy. In a surgical cricothyrotomy an incision is made in the neck, through the skin and the underlying cricothyroid membrane, to allow air to pass to the lungs (American Society of Anesthesiologists Task Force, 2003; Elliot, 1999.) Paramedics are expected to be able to manage the difficult airway and perform a surgical cricothyrotomy, yet this is extremely challenging. Even the most experienced physicians in airway management recognize the sense of urgency and anxiety associated with control of the difficult airway because patients without an adequate airway will die within minutes if they do not receive appropriate treatment (Gawande, 2001). The inability to secure an airway is the most common cause of preventable death in the pre-hospital care of injured patients (Bair, Panacek, Wisner, Bales, \& Sakes, 2003.) 
State-of-the-art, high quality 2D video was used in the 2D condition. Three views of the mannequin were provided to the remote consulting physician using digital cameras directly connected to three 21 -inch high resolution monitors. One camera was a remote-controlled pan-tilt-zoom camera that the consulting physician could control. All cameras were placed in optimal positions for our particular emergency medical situation. That is, the expert physicians determined the best locations for the three cameras to enable effective observation of the diagnosis and management of a difficult airway on the mannequin. The consulting physicians also had a full screen view of the mannequin's patient monitor showing the mannequin's heart rate, blood pressure and blood oxygen saturation rates in real time. The consulting physician observed the patient monitor and camera views in a custom built work station (Figure 2.) In addition, the paramedic also had a 2D video view of the consulting physician during the simulation. This view was located at the end of the mannequin (see Figure 1.)

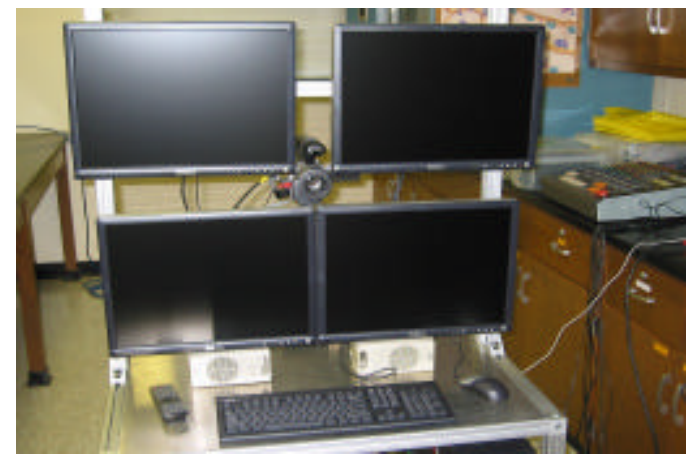

Figure 2. Consulting physician's view in the 2D condition

As mentioned earlier, the 3D telepresence technology is not yet sufficiently developed to allow us to use it even under ideal lab conditions. Therefore we designed a 3D proxy, or surrogate. For the 3D proxy condition, the consulting physician was physically present in the same room as the mannequin and paramedic. The consulting physician was allowed to freely move around in the room. However, the physician could not touch anything in the room and could only point to things using a laser pointer.

This simulates the current vision and technical goals for 3D telepresence technology (Welch et al., in press.) In this vision, remote consulting physicians will wear a small head-mounted tracking device that tracks their head movement and corresponding eye gaze. The physician's view of the accident scene will dynamically change in real time corresponding to where their eyes are currently gazing. (Alternatively the remote physician might use a hand-held tracking device to change their view of the accident scene.) This may be accomplished by using an array of cameras to capture the remote scene and computer algorithms to blend those camera views and present the result to remote physicians in real time. The goal is for remote physicians to see a 3D representation of the accident scene and be able to dynamically change their viewpoint of that scene. A remote physician will also be able to interact with the remote scene through a laser pointer that was displayed in the physician's view and at the accident scene.

\section{Study participants}

To determine the optimal number of sessions to be conducted, we reviewed the literature for similar studies and found that 10 to 20 sessions per condition was common (e.g., Gale, 1998.) It is not possible to perform a power analysis to determine the actual number needed to produce statistically significant results because we do not have an estimate for the size of the effect, or differences, between conditions. We conducted 20 sessions per each condition.

The sixty participants, 48 males and 12 females, were all professional paramedics working in southeastern US. They averaged over 7 years of experience, with a range of 1 to 26 years of experience as a certified trained paramedic. They were randomly assigned across conditions, with equal distribution of gender and years of experience over all three conditions.

Upon arrival at our facility, participants were given a hands-on introduction to the mannequin, time to 
make themselves familiar with the medical equipment available for treating the patient, and background information about the car accident that is normally available to paramedics at accident scenes.

Two emergency care physicians acted as the consulting physicians in all 2D and 3D sessions. To help reduce the impact of any individual differences these physicians may have, paramedic-physician interaction guidelines were jointly developed with these physicians. The guidelines were based on actual physician-paramedic interaction observed during the pilot study. Furthermore, each physician participated in equal numbers in both the $2 \mathrm{D}$ and $3 \mathrm{D}$ sessions.

\section{Data collection}

Each session was video-recorded using four cameras that captured paramedic's actions on and surrounding the mannequin and medical monitor output (heart rate, oxygen saturation blood levels). After a session each paramedic completed a questionnaire and participated in an open-ended interview that was audio-recorded and transcribed. Due to space limitations in this paper we focus our discussion on the questionnaire data.

\section{Evaluation Measures and Results Face validity}

Smith and Gaba (2000) define a simulation as "the artificial replication of sufficient elements of a realworld domain to achieve a specified goal" (pg. 1.) The level of accuracy of which the simulation reproduces the domain can be referred to as fidelity. The highest possible fidelity attained would be a simulation so precise that the participants in the simulation not could distinguish it from the real thing (Smith \& Gaba, 2000.)

To investigate the face fidelity of our simulation we asked participants to report their perceptions of the simulation in the post-questionnaire. In particular, 4 questions were asked (see Table 1) using a Likert scale of 1 (strongly disagree) to 7 (strongly agree.)

Table 1. Face fidelity questions and statistics

\begin{tabular}{|c|c|c|c|c|}
\hline & \multicolumn{4}{|c|}{$\begin{array}{c}\text { Mean (and SD) Questionnaire } \\
\text { Responses Scale: } 1 \text { (strongly disagree) } \\
\text { to } 7 \text { (strongly agree) }\end{array}$} \\
\hline & \multicolumn{2}{|c|}{ 2D Condition } & \multicolumn{2}{|c|}{ 3D Condition } \\
\hline & Mean & SD & Mean & SD \\
\hline I was absorbed intensely in the activity & 6.20 & .77 & 6.26 & .81 \\
\hline I concentrated fully on the activity & 6.30 & .80 & 6.35 & .75 \\
\hline The simulation was realistic & 5.75 & 1.29 & 6.20 & .83 \\
\hline $\begin{array}{l}\text { Most people would agree on how to treat the } \\
\text { patient in the simulated scenario }\end{array}$ & 5.80 & .83 & 6.30 & .80 \\
\hline Overall average scores per condition & 6.01 & .67 & 6.28 & .46 \\
\hline
\end{tabular}

In general, the participants reported they concentrated on the activity, had sufficient training, believed the simulation and medical task were realistic and doable. An analysis of variance (ANOVA) using a general linear model yielded no statistically significant differences in perceptions of fidelity due to condition, at the 0.05 level of significance. In post-interviews, participants confirmed their questionnaire responses, saying:

It felt real. [I was] absolutely absorbed. Absolutely.

It was like life.

The mannequin was awesome.

\section{Usefulness of Information}

Building on previous research by Levin and Cross (2004), usefulness of information was measured 
through 5 items on the post-questionnaire that asked to what extent the information received from the consulting physician helped or hindered key aspects of diagnosing and treating the victim in the simulation and future victims. A Likert scale was used for each question response. The scale included seven response options, including: 1 (very negatively); 2 (negatively); 3 (somewhat negatively); 4 (neither positively nor negatively); 5 (somewhat positively); 6 (positively); and, 7 (very positively.)

Table 2. Usefulness of information questions and statistics

\begin{tabular}{|c|c|c|c|c|c|c|c|}
\hline \multirow{4}{*}{$\begin{array}{l}\text { How did the information you } \\
\text { received from the physician } \\
\text { contributed to: }\end{array}$} & \multicolumn{4}{|c|}{$\begin{array}{c}\text { Questionnaire Responses } \\
\text { Scale: } 1 \text { (very negatively) to } \\
7 \text { (very positively) }\end{array}$} & & & \\
\hline & \multicolumn{2}{|c|}{ 2D Condition } & \multicolumn{2}{|c|}{ 3D Condition } & \multicolumn{3}{|c|}{ ANOVA Results } \\
\hline & Mean & SD & Mean & SD & df & $\mathbf{F}$ & $\rho$ \\
\hline & & & & & & & \\
\hline $\begin{array}{l}\text { - Diagnosing the victim } \\
\text { - Intubating the victim }\end{array}$ & $\begin{array}{l}5.68 \\
6.00\end{array}$ & $\begin{array}{l}1.11 \\
.82\end{array}$ & $\begin{array}{l}0.41 \\
6.16\end{array}$ & $\begin{array}{l}.80 \\
1.02\end{array}$ & $\begin{array}{l}1 \\
1\end{array}$ & $\begin{array}{l}5.01 \\
0.25\end{array}$ & $\begin{array}{l}.032 \\
.620\end{array}$ \\
\hline $\begin{array}{l}\text { - Performing a } \\
\text { cricothyrotomy on the } \\
\text { victim }\end{array}$ & 6.30 & .87 & 6.84 & .37 & 1 & 6.86 & .013 \\
\hline $\begin{array}{l}\text { - Increasing your } \\
\text { knowledge about } \\
\text { managing a difficult } \\
\text { airway }\end{array}$ & 5.89 & 1.15 & 6.84 & .36 & 1 & 11.66 & .002 \\
\hline $\begin{array}{l}\text { - Enhancing your future } \\
\text { performance in similar } \\
\text { situations }\end{array}$ & 6.35 & .81 & 6.84 & .38 & 1 & 5.79 & .021 \\
\hline $\begin{array}{l}\text { Overall average scores per } \\
\text { condition }\end{array}$ & 6.05 & 1.12 & 6.63 & .33 & 1 & 11.53 & .002 \\
\hline
\end{tabular}

The data were analyzed using an analysis of variance (ANOVA) test comparing means between conditions. This analysis shows that participants believed that the information received from the consulting physician under the 3D condition, the 3D proxy, was statistically significantly (at the $\rho<=.05$ level) more useful than the information received under the 2D condition (Table 2). In particular, participants' responses indicate that information provided regarding all key aspects of diagnosing and managing a difficult airway, except the intubation task, was more useful in the 3D proxy condition. We suspect this is because intubation is a task frequently performed by paramedics and thus they require little or no advice regarding this task.

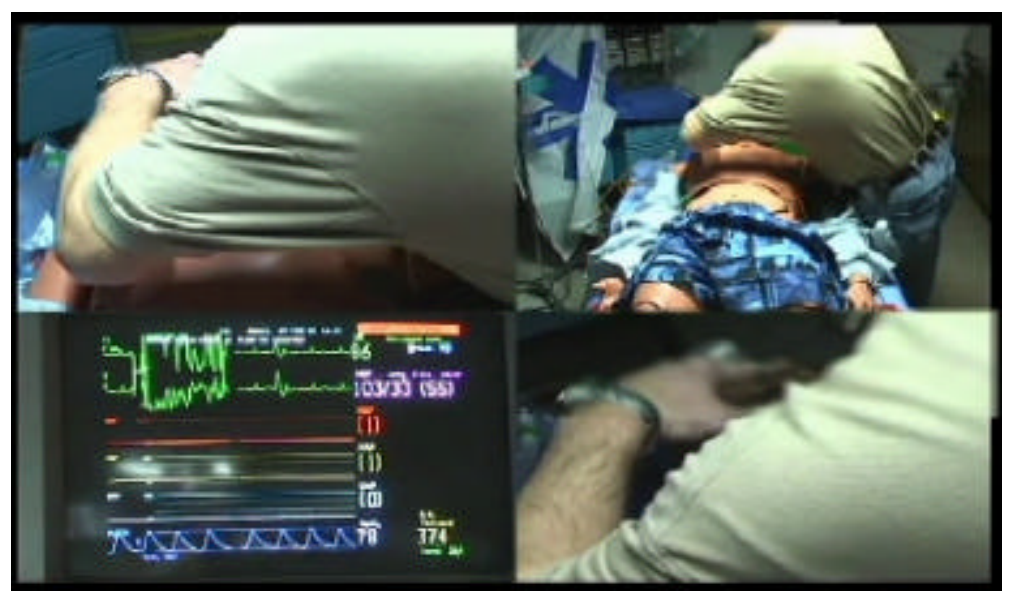

Figure 3. Typical remote physician's view in the 2D condition 
These differences may be attributed to physicians being able to more clearly see what the paramedic is doing. As mentioned previously, we followed the advice of expert physicians when positioning the cameras for the $2 \mathrm{D}$ condition. The three cameras were optimally placed for this specific scenario managing a difficult airway. In addition, one camera a pan-tilt-zoom camera that the remote physician could manoeuvre to get a different view. However, as illustrated in Figure 3 the remote physician's view is often blocked by the paramedic and medical equipment needed in the procedure. Thus the remote physician may ask questions that do not contribute to successful task completion. For example, during one session in the $2 \mathrm{D}$ condition the remote physician asked the paramedic questions such as:

Do you think your incision is big enough?

Are you in the airway?

Of course, the paramedic thinks the incision he or she has made is big enough; no paramedic would purposely make the incision too small. Similarly, a paramedic always thinks he or she has reached the airway. That is their goal; they would not purposely avoid the airway.

\section{Physician-Paramedic Interaction}

Physician-paramedic interaction was measured using four questions derived from previous research that investigated interaction between dyads mediated by information and communications technology (Short, Williams \& Christie, 1976; Chidambaram \& Jones, 1993; Sonnenwald, Maglaughlin \& Whitton, 2001). The questions focus on qualitative perceptions of the interaction because specific details can be difficult for study participants to recall.

Table 3. Physician-paramedic interaction questions and statistics

\begin{tabular}{|c|c|c|c|c|c|c|c|}
\hline \multirow{3}{*}{$\begin{array}{l}\text { Please indicate on the scale } \\
\text { the number that best describes } \\
\text { your interaction with the } \\
\text { physician: }\end{array}$} & \multicolumn{4}{|c|}{$\begin{array}{c}\text { Questionnaire Responses } \\
\text { Scale: } 1 \text { to } 7\end{array}$} & & & \\
\hline & \multicolumn{2}{|c|}{ 2D Condition } & \multicolumn{2}{|c|}{ 3D Condition } & \multicolumn{3}{|c|}{ ANOVA Results } \\
\hline & Mean & SD & Mean & SD & df & $\mathbf{F}$ & $\rho$ \\
\hline - Free (7)...Constrained (1) & 5.50 & 1.63 & 6.60 & .82 & 1 & 10.5 & .002 \\
\hline - Good (7)...Bad (1) & 6.05 & .68 & 6.50 & 1.00 & 1 & 2.41 & .129 \\
\hline - Accurate (7)...Distorted (1) & 6.40 & .68 & 6.70 & .57 & 1 & 2.28 & .139 \\
\hline - Easy (7)...Difficult (1) & 6.15 & .67 & 6.50 & .76 & 1 & 2.38 & .131 \\
\hline $\begin{array}{l}\text { Overall average scores per } \\
\text { condition }\end{array}$ & 6.03 & .73 & 6.58 & .57 & 1 & 7.07 & .011 \\
\hline
\end{tabular}

The data analysis shows that interaction between the physician and paramedic was statistically significant (at the $\rho<=.05$ level) in one dimension, i.e., free-constrained (Table 3). Participants in the 3D condition (3D proxy) evaluated their interaction with the remote physician much freer than participants in the 2D condition. In fact, all physician-paramedic interaction in the 3D condition was perceived more positively than in the 2D condition but these differences are not statistically significant. The difference with respect to the dimension of free-constrained is so profound and the other interaction items so consistent, that when combined the differences between interaction in the 2D and 3D conditions is statistically significant. Overall, interaction in the $3 \mathrm{D}$ condition is viewed more positively.

\section{Trust}

Twenty-two questions were used to measure trust. The questions were derived from previous research on trust. Butler (1991) proposes trust is comprised of ten attributes: competence, consistency, fairness, integrity, loyalty, openness, availability, promise fulfillment, receptivity, and discreetness. Butler created and validated an instrument that contained four questions for each aspect of trust plus four questions focusing on overall trust. For each aspect, three questions are positively worded and one is negatively worded. Subsequent research (Bos, Olson, Gergle, Olson, \& Wright, 2002) has used shortened versions of Butler's instrument that consist of one question for each condition and one for overall trust. We followed this approach, using 10 questions to measure each component of trust suggested by Butler (1991) and one question that focused on overall trust. 
Table 4. Trust questions and statistics

The physician acted competently (competence)

From start to finish, the physician behaved in a consistent manner

(consistency)

The physician treated me fairly (fairness)

The physician acted with integrity (integrity)

The physician did not allow other work to interfere with assisting you (loyalty)

If you gave the physician confidential information, he would keep it confidential (discreetness)

The physician had an open approach to treating the victim (openness)

It was easy to get the

physician's attention

(availability)

The physician fulfilled his professional obligations to me and the victim (promise

fulfilment)

The physician really listened to me (receptivity)

I feel that the physician can be trusted (overall trust)

I was familiar with the

terminology used by the

physician (shared language)

I could understand completely

what the physician meant when he was talking (shared

language)

It felt that we were

communicating on the same

'wavelength' (shared language)

I feel that the physician and I

care about the same issues

(shared goals)

The physician's goals are consistent and compatible with my own goals (shared goals)
Questionnaire Responses

Scale: 1 (very negatively) to 7 (very positively)

\begin{tabular}{|c|c|c|c|c|c|c|}
\hline \multicolumn{2}{|c|}{ 2D Condition } & \multicolumn{2}{|c|}{ 3D Condition } & \multicolumn{3}{|c|}{ ANOVA Results } \\
\hline Mean & SD & Mean & SD & df & $\mathbf{F}$ & $\rho$ \\
\hline 6.80 & .73 & 7.00 & 1.1 & 1 & 4.75 & .036 \\
\hline 6.85 & .37 & 6.90 & .31 & 1 & .220 & .640 \\
\hline 6.90 & .31 & 7.00 & 0 & 1 & 2.11 & .154 \\
\hline 6.84 & .36 & 7.00 & 0 & 1 & 3.56 & .067 \\
\hline 6.90 & .31 & 7.00 & 0 & 1 & 2.11 & .154 \\
\hline 6.86 & .36 & 6.89 & .32 & 1 & .070 & .796 \\
\hline 6.80 & .41 & 6.85 & .37 & 1 & .170 & .687 \\
\hline 6.79 & .47 & 6.90 & .31 & 1 & 2.53 & .120 \\
\hline 6.80 & .41 & 7.00 & 0 & 1 & 4.75 & .036 \\
\hline 6.65 & .49 & 6.95 & .22 & 1 & 6.22 & .017 \\
\hline 6.65 & .59 & 6.95 & .22 & 1 & 4.56 & .039 \\
\hline 6.79 & .54 & 6.70 & .57 & 1 & .250 & .617 \\
\hline 6.80 & .52 & 6.85 & .49 & 1 & .097 & .757 \\
\hline 6.60 & .50 & 6.75 & .44 & 1 & 1.00 & .324 \\
\hline 6.70 & .57 & 6.90 & .45 & 1 & 1.52 & .225 \\
\hline 6.75 & .55 & 6.85 & .49 & 1 & .369 & .547 \\
\hline
\end{tabular}




\section{Study Limitations}

A limitation of this study is its post-test design. Alternative evaluation experimental designs include a repeated measures and Solomon four-group design (Robson, 1997; Shadish, Cook \& Campbell, 2002). In a repeated measures, or within-subjects, design each study participant - or in our case, each pair of study participants - performs the task under all conditions. This eliminates any potential bias due to individual differences. However, it introduces a bias due to order of conditions. Study participants typically perform a task better a second time irrespective of the condition. A solution is to vary the order of the conditions and tasks in each condition. However, study participants also learn during each task session (irrespective of the condition and task) and this learning transfers to, and impacts, the second task session (e.g., see Sonnenwald, Whitton \& Maglaughlin, 2003). A Solomon four-group design in which additional groups of study participants perform different task under each condition in different order is needed to isolate such learning effects. Greater resources, in terms of numbers of study participants, time and materials, are needed to support a Solomon four-group design. Thus, we used a stratified random sampling approach and a sufficiently large number of study participants to negate potential bias due to individual differences.

A second limitation is the use of a 3D proxy instead of actual 3D telepresence technology. It is possible that the actual physical presence of the consulting physician had a significant impact on the results, and this impact may disappear with the use of 3D telepresence technology. For example, even a virtual 3D hologram of a remote consulting physician (as shown in science fiction films) may not be as effective as a face-to-face consultation. However, in emergency medical situations today paramedics interact with remote physicians over radio, and it is unclear whether face-to-face interaction makes a difference among medical professionals in emergency situations. In post-interviews, paramedics working alone and in the 2D condition reported they wanted a physician collocated to hand them instruments and to hold instruments and pieces of skin, etc. Paramedics in the 2D condition further reported they did not look at the view of the remote physician as they focused exclusively on the victim, and we noted very few instances of paramedic and physician eye-contact in either 2D or 3D sessions. However the effect of the $3 \mathrm{D}$ proxy remains an open issue which should be investigated when 3D telepresence technology is available.

\section{Discussion}

Hypothesis $\mathrm{H} 1$ that predicted paramedics would perceive the information provided by the consulting physician more useful in the 3D condition than in the 2D condition was strongly supported by the data. All information provided by the physician, except that regarding intubation, was judged to be more useful in the 3D condition. Usefulness of information is an important aspect of emergency medical care because receiving useful information has an impact not only on current task performance but also future task performance.

Hypothesis $\mathrm{H} 2$ that predicted paramedics would judge the quality of interaction with the physician as higher in the 3D condition was partially supported. The paramedics reported that their interaction was freer or less constrained in the 3D condition than in the 2D condition. However, the interaction was not necessarily better, more accurate or easier.

Hypothesis $\mathrm{H} 3$ predicted that paramedics would report greater levels of trust with respect to the consulting physician in the 3D condition as compared with the 2D condition. There is partial support for this hypothesis. In particular, the competence, promise fulfilment and receptivity of the physician, as well as overall trust towards the physician were reported statistically higher in the 3D condition. However, there was no statistical difference between conditions with respect to consistency, fairness, integrity, loyalty, discretion, openness, availability, shared language and shared goals.

Interviews we conducted with each paramedic after they completed the simulation and post-test questionnaire may provide some additional insights regarding these results. Paramedics reported that diagnosing and managing a difficult airway, including performing a surgical cricothyrotomy, are difficult to do. They reported: 
It's something I hope I don't have to do.

It's difficult making that next step, the decision that you're gonna have to do a cric.

They also reported that they appreciated having a consulting physician to assist them.

Doing it with him [the physician]...was cool..It made a big difference.

It gives you more confidence.

However, they also reported that the medical profession is hierarchical in nature. Paramedics are trained from the beginning to trust each consulting physician who has overall responsibility for the patient's care. Thus whether their interaction with the consulting physician is good or bad, accurate or distorted, easy or difficult may not be a consideration. The physician is always correct, both legally and morally.

Upon reflection, the results with respect to trust may be due to the simulation context and larger medical context. For example, during the simulation the consulting physicians followed a script that provided guidelines with respect to prompting the participating paramedic in treating the victim. The prompts were constructive in nature and included positive feedback because our expert physicians felt this is the most effective way to interact with paramedics both in the short- and long-term. Physician consistency, fairness and openness could have been impacted by this script. In addition, the physician was isolated and received no interruptions during each session, so loyalty, availability and discreetness were most likely not adequately tested during the experiment. Integrity and discreetness are hallmarks of the medical profession, and there was no or little opportunity during each session for these constructs to be invoked. Perceptions of shared language and shared goals may be attributable to a strong medical professional culture in which a highly specialized and shared language and goals exist broadly among all professionals.

The results illustrate that 3D telepresence technology shows some promise with respect to remote medical consultation in emergency medical situations. The mixed results are somewhat surprising and further investigation is needed to fully understand them. Future research includes analyzing the video recordings from each simulation session to do an in-depth analysis of task performance and interaction between paramedics and physicians in each condition.

\section{Acknowledgements}

Our sincere thanks to the study participants and to Jim Mahaney who provided expert technical assistance. This research is supported by the National Library of Medicine, contract N01-LM-3-3514: '3D Telepresence for Medical Consultation: Extending Medical Expertise Throughout, Between and Beyond Hospitals.'

\section{References}

American Society of Anesthesiologists Task Force on Difficult Airway Management (2003). Practice guidelines for management of the difficult airway. Anesthesiology, 98, 1269.1277.

Bair, A.E., Panacek, E.A., Wisner, D.H., Bales, R., \& Sakes, J.C. (2003). Cricothyrotomy: A 5-year experience at one institution. The Journal of Emergency Medicine, 24(2), 151-156.

Bos, N., Olson, J., Gergle, D., Olson, G., \& Wright, Z. (2002). Confidence and trust: Effects of four computer-mediated communications channels on trust development. Proceedings of the SIGCHI Conference on Human Factors in Computing Systems (pp. 135-140). NY: ACM Press.

Butler, J.K. (1991). Toward understanding and measuring conditions of trust: Evolution of a conditions of trust inventory. Journal of Management, 17(3), 643-663. 
Chidambaram, L., \& Jones, B. (1993). Impact of communication medium and computer support on group perceptions and performance: A comparison of faceto-face and dispersed meetings. MIS Quarterly, 17(4), 465-491.

Clarke, H. (1996). Using Language. Cambridge, UK: Cambridge University Press.

Coates, T.J., \& Goode, A. (2001). Towards improving prehospital trauma care. Lancet, 357(9274), 2070.

Dourish, P., Adler, A., Belloti, V., \& Henderson, A. (1996). Your place or mine? Learning from long-term use of audio-vide communication. Computer Supported Cooperative Work, 5 (1), 33-62.

Elliot, J.M. (1999). Airway management. In I. Greaves \& K.M. Porter (Eds.), Pre-hospital Medicine (pp. 2748). London: Arnold.

Field, M. (Ed.) (1996). Telemedicine: A guide to assessing telecommunications for health care. Washington, DC: National Academy Press.

Gale, C. (1998). The effects of gaze awareness on dialog in a video-based collaborative manipulation task. Proceedings of $\mathrm{CHI}$ '98. pp. 345-346.

Gawande., A. (2001). When doctors make mistakes. In A. Gawande, Complications: A Surgeon's Notes on an Imperfect Science (pp. 47-74). NY: Henry Holt and Company.

Levin, D.Z., \& Cross, R. (2004). The strength of weak ties you can trust: The mediating role of trust in effective knowledge transfer. Management Science, 50(11), 1477-1490.

Mayers, R.C., \& Davis, J.H. (1999). The effect of the performance appraisal system on trust for management: A field quasi-experiment. Journal of Applied Psychology, 84(1), 123-136.

Meyer, A. (1998). Death and disability from injury: A global challenge. J Trauma, 44(1), 1-12.

Olson, G.M., \& Olson, J.S. (2000). Distance matters. Human-Computer Interaction, 15(2-3), 139-178.

Olson, J.S., \& Teasley, S. (1996). Groupware in the wild: Lessons learned from a year of virtual collocation, Proceedings of the ACM 1996 conference on Computer Supported Cooperative work (pp. 419-427). NY: ACM Press.

Orlikowski, W. (1993). Learning from Notes: Organizational issues in groupware implementation, The Information Society, 9(3), 237-252.

Robson, C. (1997). Real World Research. Cambridge, MA: Blackwell.

Shadish, W., Cook, T., \& Campbell, D. (2002). Experimental and Quasi-experimental Designs for Generalized Causal Inference. NY: Houghton Mifflin.

Short, J., Williams, E., \& Christie, B. (1976). The Social Psychology of Telecommunications. NY: Wiley.

Smith, J.B., \& Barclay, D.W. (1997). The effects of organizational differences and trust on the effectiveness of selling partner relationships. Journal of Mark eting, 61, 3-21.

Smith, B., \& Gaba, D. (2000). Simulators. In C. Lake, C. Blitt, \& R. Hines (Eds.) Clinical Monitoring: Practical Application. NY: WB Saunders Company.

Sonnenwald, D.H., Maglaughlin, K.L., \& Whitton, M. C. (2001). Using innovation diffusion theory to guide collaboration technology evaluation: Work in progress. IEEE $10^{\text {th }}$ International Workshops on Enabling Technologies for Collaborative Enterprises (WET ICE). (pp. 114-119). NY: IEEE Press 
Sonnenwald, D.H., Whitton, M.C., \& Maglaughlin, K.L. (2003). Evaluating a scientific collaboratory: Results of a controlled experiment. ACM Transactions on Computer Human Interaction, (10)2, 150-176.

Star, S.L., \& Ruhleder, K. (1996). Steps toward an ecology of infrastructure: Problems of design and access in large information systems. Information Systems Research, 7, 111-134.

Welch, G., Sonnenwald, D.H., Mayer-Patel, K., Yang, R., State, A., Towles, H., Cairns, B., Maurin, H., Ilie, A., Noland, M., Noel, V., \& Fuchs, H. (in press). Remote 3D medical consultation. Journal of Mobile Multimedia. 